\title{
Prevalência de hipovitaminose D e hiperparatireoidismo secundário em mulheres na pós-menopausa
}

\author{
Prevalence of hypovitaminosis D and secondary hyperparathyroidism in \\ postmenopausal women
}

\author{
Camila Varotto Baroncini ${ }^{1}$, Thalles Pereira Regalado ${ }^{1}$, \\ Victória Zeghbi Cochenski Borba ${ }^{2}$, Carolina Aguiar Moreira ${ }^{3}$
}

Baroncini CV, Regalado TP, Borba VZC, Moreira CA. Prevalência de hipovitaminose D e hiperparatireoidismo secundário em mulheres na pós-menopausa / Prevalence of hypovitaminosis D and secondary hyperparathyroidism in postmenopausal women. Rev Med (São Paulo). 2018 jul.-ago.;97(4):378-84.

RESUMO: Introdução: A vitamina D e o paratormônio (PTH) são os principais moduladores do metabolismo mineral, contribuindo para homeostase do cálcio e fósforo. Considera-se como insuficientes níveis séricos abaixo de $30 \mathrm{ng} / \mathrm{mL}$ de 25 -hidroxivitamina $\mathrm{D}[25(\mathrm{OH}) \mathrm{D}]$ e deficientes quando abaixo de $20 \mathrm{ng} /$ $\mathrm{mL}$. Tal deficiência também ocasiona aumento da secreção de PTH e, assim, há aumento da desmineralização óssea. Este estudo visa avaliar os níveis séricos de $25(\mathrm{OH}) \mathrm{D}$ em mulheres na pós-menopausa e correlacioná-los com as concentrações de PTH e a densidade mineral óssea (DMO). Materiais e métodos: Foram coletados dados clínicos, laboratoriais e densitométricos de 114 mulheres na pós-menopausa que participaram de estudo clínico prévio. As pacientes incluídas apresentavam diagnóstico de osteopenia ou osteoporose sem tratamento prévio. A análise estatística foi realizada pelo software Statistica - Statsoft ${ }^{\circledR}$. Resultados: As pacientes foram classificadas em osteoporóticas $82,9 \%(\mathrm{n}=92)$ e osteopênicas $17,1 \%(\mathrm{n}=19)$. A média dos valores de $25(\mathrm{OH}) \mathrm{D}$ encontrada foi $26,21 \pm 9,69 \mathrm{ng} / \mathrm{mL}$. Identificou-se deficiência $(16,05 \pm 2,81 \mathrm{ng} / \mathrm{mL})$ em $32,4 \%$ das pacientes $(\mathrm{n}=$ $37)$ e insuficiência $(24,71 \pm 3,18 \mathrm{ng} / \mathrm{mL})$ em $33,3 \%(\mathrm{n}=38)$. Os valores de PTH apresentaram média de 53,7 $\pm 20,1 \mathrm{pg} / \mathrm{mL}$. Destes, $25 \%(n=29)$ apresentaram-se acima dos valores de referência.
Os valores séricos de PTH correlacionaram-se negativamente com os valores séricos de 25(OH)D $(\mathrm{p}<0,01$; rho $=-0.2971)$. Houve uma correlação negativa do T-score de colo de fêmur em duas situações: histórico de fraturas e no diagnóstico de fraturas de coluna $(\mathrm{p}<0,01)$. Conclusão: Neste grupo de mulheres pósmenopausadas houve uma alta prevalência de hipovitaminose $\mathrm{D}$, a qual se correlacionou negativamente com o PTH, não sendo observado relação com as estações do ano. Houve uma correlação entre a história de fraturas e a DMO, evidenciando que a DMO de fêmur pode predizer risco de fraturas em outros sítios.

Descritores: Deficiência de vitamina D; Hormônio paratireóideo; Doenças ósseas metabólicas; Pós-menopausa.

\begin{abstract}
Introduction: Vitamin D and parathyroid hormone (PTH) are two major modulators of mineral metabolism and the maintenance of calcium and phosphorus homeostasis. Vitamin $\mathrm{D}$ insufficiency is characterized by serum 25 -hydroxy-vitamin $\mathrm{D}$ [25(OH)D] concentration under $30 \mathrm{ng} / \mathrm{mL}$ and deficiency under $20 \mathrm{ng} / \mathrm{mL}$. Such deficiency also increases PTH secretion and bone demineralization. This study aims to evaluate serum $25(\mathrm{OH}) \mathrm{D}$ concentrations in postmenopausal women and correlate them
\end{abstract}

Universidade Federal do Paraná (UFPR). Serviço de Endocrinologia e Metabologia do Paraná - parte do Complexo Hospital de Clínicas UFPR. Apresentação oral como Trabalhos do Curso de Medicina da UFPR, 5-11 nov. 2017. Pôster na Jornada de Clínica Médica do Hospital de Clínicas UFPR, 30 set. 2017.

1. Acadêmicos do $5^{\circ}$ ano de Medicina da Universidade Federal do Paraná. E-mail: camilavbaroncini@gmail.com, thallesregalado@gmail.com.

2.Professora do Departamento de Clínica Médica da Universidade Federal do Paraná. Médica da Unidade de Metabolismo Ósseo do Serviço de Endocrinologia e Metabologia da UFPR - SEMPR. E-mail: vzcborba@gmail.com.

3. Professora do Departamento de Clínica Médica da Universidade Federal do Paraná. Médica da Unidade de Metabolismo Ósseo do Serviço de Endocrinologia e Metabologia da UFPR - SEMPR. Médica do Serviço de Densitometria Óssea do Complexo Hospital de Clínicas. E-mail: carolina.aguiar.moreira@gmail.com.

Autor para correspondência: Carolina Aguiar Moreira. Av. Agostinho Leão Junior, 285. Alto da Giria. Curitiba, PR, Brasil. CEP: 800030-110. Email: carolina.aguiar.moreira@gmail.com. 
Baroncini CV, et al. Prevalência de hipovitaminose D e hiperparatireoidismo secundário em mulheres.

with serum PTH concentrations and bone mineral density (BMD). Methods: Clinical, laboratorial and densitometric data were collected from 114 postmenopausal women who participated in a previous clinical study. The patients recruited had a diagnosis of osteopenia or osteoporosis without previous treatment. Statistical analysis was carried out by the Statistica-Statsoft $\AA$ software. Results: Patients were divided in osteoporotic $82.9 \%(\mathrm{n}=92)$ and osteopenic $17.1 \%(n=19)$. The mean values of $25(\mathrm{OH}) \mathrm{D}$ found were $26.21 \pm 9.69 \mathrm{ng} / \mathrm{mL}$. Deficiency $(16.05 \pm 2.81 \mathrm{ng} / \mathrm{mL})$ was identified in $32,4 \%$ of patients $(n=37)$ and insufficiency $(24.71$ $\pm 3.18 \mathrm{ng} / \mathrm{mL})$ in $33,3 \%(\mathrm{n}=38)$. PTH presented a mean of 53.7 $\pm 20.1 \mathrm{pg} / \mathrm{mL}$ and $25 \%(\mathrm{n}=29)$ were above the reference values.

\section{INTRODUÇÃO}

vitamina D e o paratormônio (PTH) apresentam papel fundamental para o metabolismo ósseo ${ }^{1}$. Ambos, por meio de um controlado sistema de feedback, contribuem para a homeostase de cálcio e fósforo no organismo. Os baixos níveis séricos de cálcio estimulam a liberação de PTH pelas glândulas paratireoides e este age no esqueleto, liberando cálcio ionizado $(\mathrm{Ca} 2+)$ e fosfato (HPO42-) na circulação sanguínea. Nos rins, o PTH estimula a reabsorção de $\mathrm{Ca} 2+$ e diminui a reabsorção de HPO42-, ao mesmo tempo em que exerce feedback positivo para a síntese de vitamina $D$. Esta, por sua vez, age favorecendo a absorção intestinal de cálcio e contribuindo para a formação óssea adequada. A restauração de níveis normais de cálcio sérico aliada às concentrações adequadas de vitamina D têm efeito inibitório na secreção de $\mathrm{PTH}^{1}$. Assim, ambos hormônios têm impacto sobre a densidade óssea e a prevenção de fraturas osteoporóticas ${ }^{2}$.

Atualmente, há uma discussão em relação aos valores séricos de referência da $25(\mathrm{OH}) \mathrm{D}$. A Sociedade Brasileira de Endocrinologia e Metabologia propõe que para a população saudável, valores $>20 \mathrm{ng} / \mathrm{mL}$ são adequados e que para os pacientes com fatores de risco para osteoporose, valores superiores a $30 \mathrm{ng} / \mathrm{mL}$ são necessários ${ }^{3,4}$. Importante ressaltar que o nível esperado de $25(\mathrm{OH}) \mathrm{D}$ seria aquele necessário para manter o $\mathrm{PTH}$ em níveis normais e evitar as consequências ósseas do hiperparatireoidismo secundário 5 .

Entretanto, existe uma epidemia de hipovitaminose $\mathrm{D}$ bem documentada na literatura mundial ${ }^{6}$. A deficiência de vitamina D é comum em idosos e em pacientes com osteoporose e correlaciona-se com perda de massa óssea e aumento do risco de quedas e, consequentemente, fraturas ${ }^{7}$.

Este estudo, portanto, teve como finalidade avaliar a prevalência de hipovitaminose $\mathrm{D}$ em um grupo de mulheres que foram atendidas na Unidade de Metabolismo Ósseo do Serviço de Endocrinologia e Metabologia do HC da UFPR (SEMPR) e correlacionar os valores da $25(\mathrm{OH}) \mathrm{D}$ com os níveis séricos de PTH e a densidade mineral óssea.

\section{MATERIAIS E MÉTODOS}

Estudo observacional retrospectivo transversal, no
Concentrations of PTH correlated inversely with 25(OH)D (p < $0,01 ;$ rho $=-0,2971)$. There was a negative correlation of femoral neck T-score in two situations: history of fractures and diagnosis of spinal fractures $(p<0,01)$. Conclusion: Hypovitaminosis D has been shown to be prevalent among these post menopausal women with no seasonal influence. There was a negative correlation between PTH levels and 25(OH)D. A correlation between the history of fractures and BMD was found, therefore, femur BMD can predict risk of fractures in other sites.

Keywords: Vitamin D Deficiency. Parathyroid hormone; Bone diseases, metabolic; Postmenopause.

qual os prontuários de pacientes que participaram de uma seleção para um estudo clínico realizado no período de 2011 a 2012 no SEMPR, foram revisados.

Os critérios de inclusão tanto para o estudo clínico, como para o presente estudo, foram:

a) sexo feminino acima de 50 anos na pós menopausa (ausência de menstruação há pelo menos 24 meses);

b) osteopenia ou osteoporose detectadas pela densitometria óssea (osteopenia entre -1,1 e 2,4 e osteoporose abaixo de $-2,5$ de escore T).

Como critérios de exclusão foram considerados:

a) pacientes com outras doenças osteometabólicas ou antecedentes de uso de medicação que interferem no metabolismo ósseo (ex.: corticosteróides, anticonvulsivantes, metotrexato);

b) pacientes que receberam tratamento prévio para osteoporose/osteopenia ou reposição de vitamina D.

Os dados coletados abrangeram as características intrínsecas às pacientes [idade, peso, altura, índice de massa corporal (IMC) e raça], o histórico de fraturas atraumáticas (localização e quantidade) definido pela anamnese, os dados laboratoriais (PTH sérico intacto, $25(\mathrm{OH}) \mathrm{D}$ sérica, fosfato sérico, cálcio sérico, cálcio total corrigido pela albumina, cálcio urinário, creatinina sérica e cleareance urinário), a densidade mineral óssea (DMO) de fêmur total, colo de fêmur e coluna lombar e a presença ou não de microfraturas em radiografia de coluna de controle. As datas das coletas de sangue para dosagem da $25(\mathrm{OH}) \mathrm{D}$ e realização dos exames foram anotadas e classificados de acordo com a estação do ano. Todos os dados foram registrados pelos pesquisadores em planilha eletrônica (Microsoft Excel ${ }^{\circledR}$ ).

A análise estatística foi realizada por meio do software (Statistica - Statsoft $\AA$ ). Foram calculados a média, o desvio-padrão, máxima, mínima e mediana das variáveis quantitativas. As pacientes foram classificadas, separadamente, de acordo com a densitometria óssea e os níveis séricos de $25(\mathrm{OH}) \mathrm{D}$ e PTH. Os testes estatísticos foram realizados conforme a natureza das variáveis e tipos de análises necessárias. A análise de correlação de Spearman foi empregada para avaliar a correlação entre as concentrações de $25(\mathrm{OH}) \mathrm{D}$ e PTH, e de cada um deles com os dados laboratoriais e densitométricos. O teste de Kruskal Wallis foi utilizado para verificar PTH e $25(\mathrm{OH})$ $\mathrm{D}$ em relação às variáveis qualitativas. Os testes exato 
de Fisher e Qui-quadrado correlacionaram as variáveis qualitativas entre si. Os testes t de student e Mann-Whitney correlacionaram as variáveis quantitativas de distribuição normal (IMC, idade, PTH, 25(OH)D e os demais dados laboratoriais e densitométricos) com a presença de fraturas e classificação da DMO em osteopenia/osteoporose cada variável foi analisada separadamente. O nível de significância foi considerado de $5 \%$ e a amostra calculada para obter poder de teste mínimo de $90 \%$.

\section{RESULTADOS}

Foram selecionadas 114 mulheres, todas eram caucasianas. A média de idade observada foi 70,4 $\pm 6,81$ anos e o IMC médio $26,32 \pm 3,71 \mathrm{~kg} / \mathrm{m}^{2}$. Os dados laboratoriais estão demonstrados na Tabela 1. Todas as pacientes apresentavam redução da DMO e foram classificadas em osteoporóticas $82,5 \%(\mathrm{n}=94)$ e osteopênicas $17,5 \%(n=20)$.

Tabela 1 - Dados antropométricos e laboratoriais

\begin{tabular}{l|c}
\hline Variáveis & Média \pm Desvio-padrão \\
\hline Idade (anos) & $70,4 \pm 6,81$ \\
IMC $\left(\mathrm{kg} / \mathrm{m}^{2}\right)$ & $26,3 \pm 3,71$ \\
PTH $(\mathrm{pg} / \mathrm{mL})$ & $53,7 \pm 20,1$ \\
25(OH)D $(\mathrm{ng} / \mathrm{mL})$ & $26,2 \pm 9,69$ \\
Cálcio sérico corrigido (mmol/L) & $2,29 \pm 0,09$ \\
Cálcio urinário (mg/dL) & $2,32 \pm 1,77$ \\
Fosfato sérico $(\mathrm{mmol} / \mathrm{L})$ & $1,19 \pm 0,13$ \\
Creatinina sérica $(\mathrm{mg} / \mathrm{dL})$ & $0,69 \pm 0,16$ \\
Clearance urinário $(\mathrm{mL} / \mathrm{min})$ & $76,1 \pm 28,6$ \\
\hline
\end{tabular}

FONTE: Os autores (2017).

Os exames foram realizados majoritariamente no outono $(n=44 ; 38,6 \%)$ e inverno $(n=43 ; 37,7 \%)$. Não houve correlação estatisticamente significativa entre as estações do ano e os valores séricos de PTH $(\mathrm{p}=0,332)$, $25(\mathrm{OH}) \mathrm{D}(\mathrm{p}=0,338)$ e com o valor de T-score encontrados na DMO.

A média dos valores de 25(OH)D encontrada foi $26,21 \pm 9,69 \mathrm{ng} / \mathrm{mL}$. Trinta e dois por cento das pacientes ( $\mathrm{n}=37)$ apresentaram deficiência de vitamina $\mathrm{D}(16,05$ $\pm 2,81 \mathrm{ng} / \mathrm{mL}), 33 \%(\mathrm{n}=38)$ insuficiência $(24,71 \pm 3,18$ $\mathrm{ng} / \mathrm{mL})$ e $34 \%(\mathrm{n}=39)$ tinham níveis adequados $(37,39 \pm$ $5,79 \mathrm{ng} / \mathrm{mL}$ ), como ilustrado no Gráfico 1. As correlações de Spearman calculadas estão demonstradas na Tabela 2.
Gráfico 1 - Concentrações de 25(OH)D

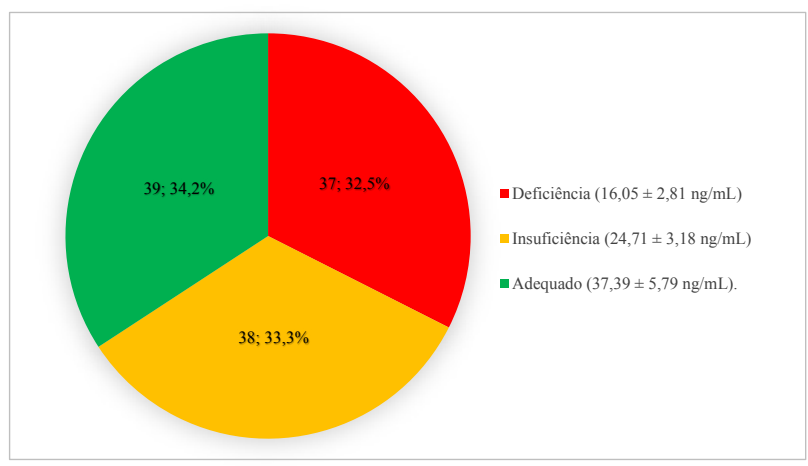

FONTE: Os autores, 2017.

Tabela 2 - Correlação entre 25(OH)D e os diversos parâmetros avaliados

\begin{tabular}{l|l}
\hline Parâmetro & valor $\mathbf{p}$ \\
\hline Idade $(\mathrm{anos})$ & 0,3888 \\
$\mathrm{IMC}\left(\mathrm{kg} / \mathrm{m}^{2}\right)$ & 0,1223 \\
PTH $(\mathrm{pg} / \mathrm{mL})$ & 0,$0013 ;$ rho $=-0,297$ \\
Cálcio sérico corrigido $(\mathrm{mmol} / \mathrm{L})$ & 0,2986 \\
Cálcio urinário $(\mathrm{mmol} / \mathrm{L})$ & 0,5857 \\
Fosfato sérico $(\mathrm{mmol} / \mathrm{L})$ & 0,1289 \\
Clearance urinário $(\mathrm{mL} / \mathrm{min})$ & 0,5861 \\
T-score de L1-L4 & 0,2269 \\
T-score de colo de fêmur & 0,3017 \\
T-score de fêmur total & 0,9273 \\
\hline
\end{tabular}

FONTE: Os autores, 2017.

Os valores de PTH observados tinham média de $53,7 \pm 20,1 \mathrm{pg} / \mathrm{mL}$. Vinte e cinco por cento das pacientes $(\mathrm{n}=29)$ apresentaram PTH acima do valor de referência (10-65 pg/mL ou $10-65 \mathrm{ng} / \mathrm{L})$. Houve uma correlação negativa entre os valores séricos de PTH com os valores de 25(OH)D ( $p<0,01 ;$ rho $=-0,2971)$, como demonstrado na Figura 1. Ao analisar, por meio do teste de comparações múltiplas, o PTH sérico nos grupos de pacientes com 25(OH)D normal, insuficiente ou deficiente, a diferença é estatisticamente significativa entre o grupo com vitamina normal e deficiente. Não houve diferença na comparação entre o grupo normal com o grupo insuficiente e entre o grupo insuficiente com o grupo deficiente.

Outros dados laboratoriais e suas correlações estão representados na Tabela 3. O único com correlação inversa significativa foi o fosfato sérico $(p<0,01$; rho $=-0,28)$, como era de se esperar pela fisiologia do PTH.

A idade não se correlacionou significativamente com as concentrações de PTH e 25(OH)D. No entanto, houve correlação negativa significativa com os T-scores de colo de fêmur $(\mathrm{p}<0,05$; rho $=-0,225)$ e fêmur total $(\mathrm{p}<$ $0,05 ;$ rho $=-0,189)$. Mulheres com idades mais avançadas apresentaram maior tendência a apresentar microfraturas à radiografia de coluna, de acordo com $o$ teste $t$ de student $(\mathrm{p}<0,001)$. 


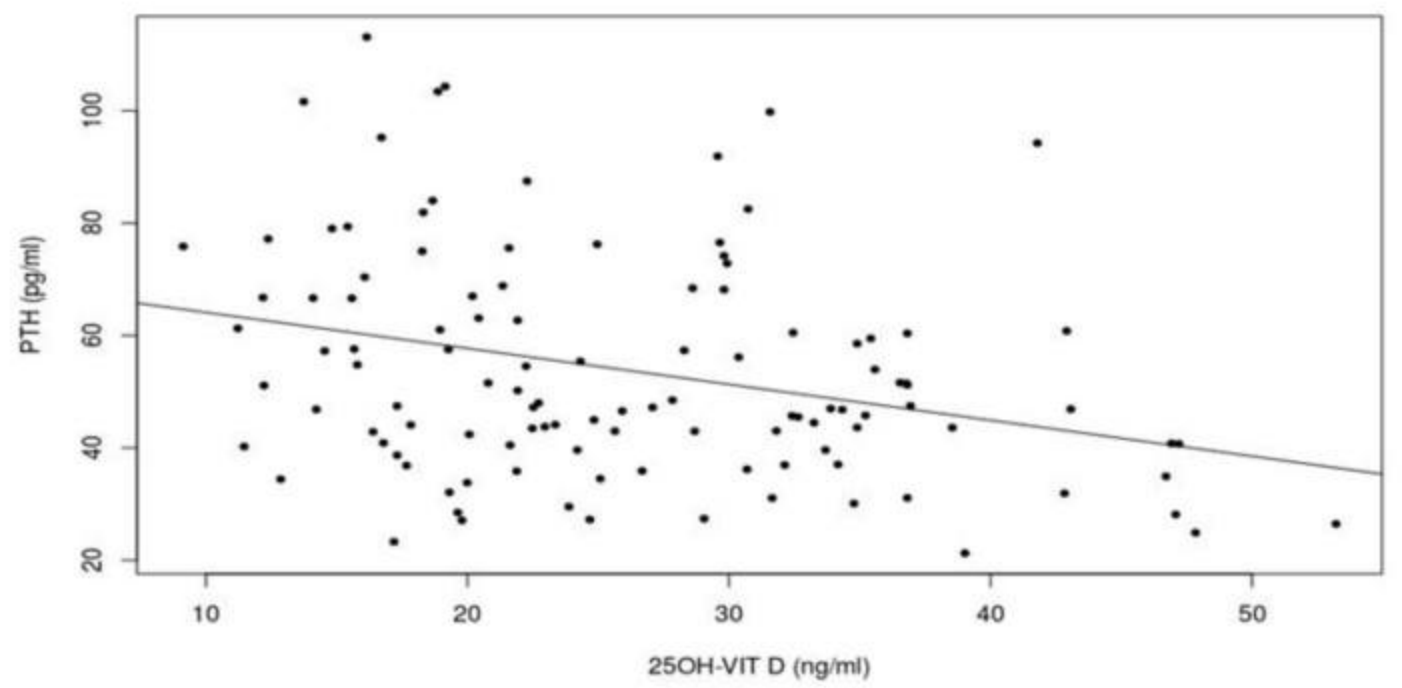

Teste: Correlação de Spermann $(p=0,0013 ;$ rho = -0,2971)

FONTE: Os autores, 2017.

Figura 1 - Correlação entre os níveis de PTH E 25(OH)D

Tabela 3 - Correlação entre PTH e os diversos parâmetros avaliados

\begin{tabular}{l|l}
\hline Parâmetro & valor p \\
\hline Idade $(\mathrm{anos})$ & 0,699 \\
$\mathrm{IMC}\left(\mathrm{kg} / \mathrm{m}^{2}\right)$ & 0,517 \\
Cálcio sérico corrigido $(\mathrm{mmol} / \mathrm{L})$ & 0,245 \\
Cálcio urinário $(\mathrm{mmol} / \mathrm{L})$ & 0,153 \\
Fosfato sérico $(\mathrm{mmol} / \mathrm{L})$ & 0,$028 ;$ rho $=-0,277$ \\
Clearance urinário $(\mathrm{mL} / \mathrm{min})$ & 0,409 \\
T-score de L1-L4 & 0,167 \\
T-score de colo de fêmur & 0,592 \\
T-score de fêmur total & 0,407 \\
\hline
\end{tabular}

FONTE: Os autores, 2017.

Quanto à DMO, os valores encontrados estão apresentados na Tabela 4. Também de acordo com o coeficiente de Spearman, o IMC correlacionou-se positivamente com a DMO da L1-L4 $(\mathrm{p}<0,05$ e rho $=$ 0,29 ; colo de fêmur: $\mathrm{p}<0,001$ e rho $=0,312$; fêmur total: $\mathrm{p}<0,001$ e rho $=0,533)$. Evidenciou-se pelo teste t-student que pacientes osteoporóticos apresentam IMC menor que os pacientes com osteopenia ( $p<0,01)$. Não houve correlação entre DMOs de coluna lombar e fêmur com as concentrações séricas de 25(OH)D e PTH.
Tabela 4 - Dados densitométricos

\begin{tabular}{l|l}
\hline Variáveis & Média \pm Desvio-padrão \\
\hline DMO L1-L4 $\left(\mathrm{g} / \mathrm{cm}^{2}\right)$ & $0,812 \pm 0,098$ \\
T-score L1-L4 & $-2,941 \pm 0,845$ \\
DMO Colo de fêmur $\left(\mathrm{g} / \mathrm{cm}^{2}\right)$ & $0,724 \pm 0,091$ \\
T-score Colo de fêmur & $-2,195 \pm 0,828$ \\
DMO Fêmur Total $\left(\mathrm{g} / \mathrm{cm}^{2}\right)$ & $0,756 \pm 0,108$ \\
T-score Fêmur Total & $-1,934 \pm 0,934$ \\
\hline
\end{tabular}

FONTE: Os autores, 2017.

A história de fraturas atraumáticas foi positiva em $70,8 \%$ das pacientes $(\mathrm{n}=80)$. A localização mais frequente foi a de antebraço em $37 \%(\mathrm{n}=32)$ das pacientes; seguida pela de membro inferior $(22,8 \%, \mathrm{n}=26)$ e quadril $(9,6 \%$, $\mathrm{n}=11)$. Evidenciou-se que pacientes com fraturas prévias apresentaram T-score de coluna lombar mais baixo que as pacientes com histórico negativo para fraturas, entretanto, isso não foi significativo para os T-scores de colo de fêmur e fêmur total. A relação do histórico de fraturas com a classificação de pacientes entre osteoporóticas e osteopênicas não foi significativa $(p=0,06)$, assim como a correlação com $25(\mathrm{OH}) \mathrm{D}(\mathrm{p}=0,39)$ e PTH séricos $(\mathrm{p}=0,39)$.

Foi observada uma prevalência de $27 \%(n=30)$ de fraturas morfométricas avaliadas na radiografia de coluna de controle realizada. Um maior número de fraturas atraumáticas foi identificado com o aumento da idade $(\mathrm{p}<$ $0,01)$. Além disto, as pacientes que apresentavam fraturas morfométricas ao raio $\mathrm{x}$, tinham um menor T-score do colo de fêmur. 


\section{DISCUSSÃO}

No presente estudo, demonstrou-se uma prevalência elevada de hipovitaminose D em um grupo de mulheres procedentes de Curitiba, o que está de acordo com a literatura internacional e nacional. Além disto, foi observada uma correlação negativa com PTH. Em um estudo global ${ }^{8}$, com 26 países, relatou-se $28,4 \%$ de deficiência da vitamina $D$ numa população de 7564 mulheres na pós-menopausa $(<20$ $\mathrm{ng} / \mathrm{mL}$ ). Já a porcentagem de mulheres na pós-menopausa com osteoporose que apresentam 25(OH)D sérica $<30$ $\mathrm{ng} / \mathrm{mL}$ (insuficiência) durante o inverno aproxima-se de 90-100\% na Europa e 80\% no Canadá e Estados Unidos 9 . Mesmo no Brasil, país com melhor status de 25(OH)D no estudo multicêntrico, apenas $34,3 \%$ das mulheres possuíam níveis séricos adequados no inverno e $43 \%$ no verão 9 . Em um estudo realizado em São Paulo, foi demonstrada uma alta prevalência de insuficiência $(41,9 \%)$ e deficiência $(15,4 \%)$ de vitamina D associada ao hiperparatireoidismo secundário (55\%) em uma população sem fatores de risco ${ }^{10}$. Em Belo Horizonte, numa pesquisa realizada com 180 pacientes ambulatoriais, encontrou-se uma prevalência de $42,4 \%$ de deficiência de vitamina $\mathrm{D}^{11}$.

Os principais fatores que contribuem para a insuficiência ou deficiência de vitamina D são a baixa exposição solar, presença de doenças ou drogas que afetam seu metabolismo e a dieta pobre em vitamina $\mathrm{D}^{12,13}$. Em nosso estudo, não foi abordado a dieta das pacientes quanto ingesta de cálcio e vitamina $\mathrm{D}$, mas causas secundárias que poderiam afetar as dosagens de vitamina $\mathrm{D}$ foram excluídas. As prováveis causas da população geriátrica ser mais suscetível à hipovitaminose $\mathrm{D}$ parecem se relacionar ao fato de o grupo sofrer menor exposição solar, ter sua capacidade de produção de $25(\mathrm{OH}) \mathrm{D}$ reduzida (principalmente por apresentar função renal diminuída), alimentar-se de forma inadequada, absorver menos vitamina pelo trato gastrointestinal, além de usar múltiplas drogas que interferem na sua absorção e metabolização ${ }^{13,14}$.

Sabe-se que as estações do ano também parecem exercer efeito nas concentrações de vitamina $\mathrm{D}$, principalmente em regiões de clima temperado ${ }^{13,15-20}$. Em nossa pesquisa, não foi observado esta associação provavelmente pelo fato da maioria das pacientes terem realizado a coleta para avaliação de $25(\mathrm{OH}) \mathrm{D}$ nas estações de outono e inverno, apresentando uma falta de dispersão da amostra.

A prevalência de hiperparatireoidismo secundário a hipovitaminose D nesta amostra foi de $25 \%$, diferente de outros estudos brasileiros que observaram uma prevalência superior a $60 \%$, o que provavelmente se deve ao fato dos pacientes serem mais idosos do que as pacientes deste estudo. Saraiva ${ }^{10}$ apresentou $66 \%$ de prevalência de hipovitaminose $\mathrm{D}$ em uma população com mais de 65 anos, tendo $55 \%$ de hiperparatireoidismo secundário associado. Em 2007, numa comparação entre pacientes institucionalizados e ambulatoriais, o hiperparatiroidismo secundário ocorreu em $61,7 \%$ dos pacientes do grupo institucionalizado e em $54 \%$ dos pacientes do grupo ambulatorial ${ }^{21}$. Entretanto, nossos dados demonstraram correlação negativa significativa de 25(OH)D e PTH, em concordância com outros estudos, apesar de diferentes valores de coeficiente ${ }^{13,22-25}$. Esta correlação, a qual é mais evidente quando o nível da 25(OH)D está abaixo de $30 \mathrm{ng} /$ $\mathrm{mL}$, reforça que o valor ideal da $25 \mathrm{OHD}$ para pacientes com osteoporose, seja superior a $30 \mathrm{ng} / \mathrm{ml}^{9,13}$.

O IMC correlacionou-se positivamente com a DMO, sendo os pacientes com osteoporose apresentavam um IMC significativamente menor do que aqueles com osteopenia. Esses dados são concordantes com a literatura ${ }^{26-29}$, que indica que o excesso de peso, tanto devido a gordura corporal como pelos músculos esqueléticos, estimula a osteogênese ao exercer força mecânica sobre os ossos ${ }^{30-31}$.

Evidenciou-se que pacientes com fraturas prévias apresentaram T-score de L1-L4 mais baixo que as pacientes com histórico negativo para fraturas, entretanto, isso não foi significativo para os T-scores de colo de fêmur e fêmur total. Essa associação da história de fraturas com a DMO também já foi documentada ${ }^{32-34}$.

Em relação a microfraturas na radiografia de coluna presentes em 1/3 das pacientes, não foram correlacionadas com a concentração de vitamina $\mathrm{D}$; entretanto, quase metade dessas pacientes apresentou concentrações de $25(\mathrm{OH}) \mathrm{D}<30 \mathrm{ng} / \mathrm{mL}$. Este resultado é similar ao encontrado em um estudo realizado no Rio de Janeiro ${ }^{22}$, que identificou fraturas vertebrais assintomáticas em 20\% das mulheres na pós-menopausa investigadas, sendo que $57,7 \%$ delas eram de pacientes deficientes ou insuficientes em vitamina $\mathrm{D}$. Apesar disso, também não foi apontado correlação significativa com a 25(OH)D sérica. Em nossa série, as fraturas atraumáticas foram correlacionadas com o aumento da idade e com um menor valor de T-score do colo de fêmur, resultados estes concordantes com a literatura.

Entretanto, nosso estudo possui limitações. A amostra não foi representativa da população geral, visto que a seleção foi restrita às mulheres com DMO alterada. Faltou um grupo controle, pacientes sem osteopenia e osteoporose, para melhor avaliação dos dados.

\section{CONCLUSÃo}

Concluimos que foi observado uma elevada prevalência de hipovitaminose D nesta amostra de pacientes, mulheres provenientes de Curitiba, sul do Brasil, em pós-menopausa com DMO baixa. Os valores da vitamina D se correlacionaram negativamente com as concentrações de PTH, semelhante a outros estudos da literatura.

Evidenciou-se também uma correlação entre a história de fraturas, como também fraturas morfométricas, com a DMO, comprovando o valor preditivo para fraturas 
Baroncini CV, et al. Prevalência de hipovitaminose D e hiperparatireoidismo secundário em mulheres.

do exame de densitometria óssea.

Com estes achados, reforçamos a importância da suficiência da vitamina D no manejo das pacientes com

\section{REFERÊNCIAS}

1. Khundmiri SJ, Murray RD, Lederer E. PTH and vitamin D. Compr Physiol. 2016;6(2):561-601. doi: 10.1002/cphy. c140071.

2. Reginster J-Y. Calcium and vitamin D for osteoporotic fracture risk. Lancet. 2007;370:632-4. doi: 10.1016/S01406736(07)61315-4.

3. Lips P. Which circulating level of 25-hydroxyvitamin D is appropriate? J Steroid Biochem Mol Biol. 2004;89-90(15):611-4. doi: 10.1016/S0140-6736(07)61315-4.

4. Dawson-Hughes B, Heaney RP, Holick MF, Lips P, Meunier PJ, Vieth R. Estimates of optimal vitamin D status. Osteoporos Int. 2005;16(7):713-6. doi: 10.1007/s00198-005-1867-7.

5. Castro LCG de. O sistema endocrinológico vitamina D. Arq Bras Endocrinol Metabol. 2011;55(8):566-75. http://dx.doi. org/10.1590/S0004-27302011000800010.

6. Souberbielle J-C, Body J-J, Lappe JM, Plebani M, Shoenfeld Y, Wang TJ, et al. Vitamin D and musculoskeletal health, cardiovascular disease, autoimmunity and cancer: Recommendations for clinical practice. Autoimmun Rev. 2010;9(11):709-15. doi: 10.1016/j.autrev.2010.06.009.

7. Lips P, Hosking D, Lippuner K, Norquist JM, Wehren L, Maalouf G, et al. The prevalence of vitamin D inadequacy amongst women with osteoporosis: an international epidemiological investigation. J Intern Med. 2006;260(3):24554. doi: 10.1111/j.1365-2796.2006.01685.x.

8. Lips P, Duong T, Oleksik A, Black D, Cummings S, Cox D, et al. A global study of vitamin d status and parathyroid function in postmenopausal women with osteoporosis: baseline data from the Multiple Outcomes of Raloxifene Evaluation Clinical Trial. J Clin Endocrinol Metab. 2001;86(3):1212-21. doi: $10.1210 /$ jcem.86.3.7327.

9. Kuchuk NO, van Schoor NM, Pluijm SM, Chines A, Lips P. Vitamin D status, parathyroid function, bone turnover, and BMD in postmenopausal women with osteoporosis: global perspective. J Bone Miner Res. 2009;24(4):693-701. doi: 10.1359/jbmr.081209

10. Saraiva GL, Cendoroglo MS, Ramos LR, Araújo LMQ, Vieira $\mathrm{JGH}, \mathrm{Kunii}$ I, et al. Influence of ultraviolet radiation on the production of 25 hydroxyvitamin $\mathrm{D}$ in the elderly population in the city of São Paulo (23 degrees, 34'S), Brazil. Osteoporos Int. 2005;16(12):1649-54. doi: 10.1007/s00198-005-1895-3

11. Silva BCC, Camargos BM, Fujii JB, Dias EP, Soares MMS. Prevalência de deficiência e insuficiência de vitamina D e sua correlação com PTH, marcadores de remodelação óssea e densidade mineral óssea, em pacientes ambulatoriais. Arq Bras Endocrinol Metabol. 2008;52(3):482-8. doi: 10.1590/ S0004-27302008000300008.

12. Holick MF, Chen TC, Lu Z, Sauter E. Vitamin D and skin physiology: A D-lightful story. J Bone Miner Res. 2007;22(S2):V28-33. doi: 10.1359/jbmr.07s211.

13. Maeda SS, Saraiva GL, Hayashi LF, Cendoroglo MS, Ramos baixa densidade óssea, o que contribuirá na prevenção do hiperparatireoidismo secundário e no aumento do risco de fraturas.

LR, Corrêa M de P, et al. Seasonal variation in the serum 25-hydroxyvitamin D levels of young and elderly active and inactive adults in São Paulo, Brazil. Dermatoendocrinol. 2013;5(1):211-7. doi: 10.4161/derm.24476.

14. Russo LAT, Gregório LH de, Lacativa PGS, Marinheiro LPF. Concentração plasmática de 25 hidroxivitamina D em mulheres na pós-menopausa com baixa densidade mineral óssea TT - concentration of 25-hydroxyvitamin D in postmenopausal women with low bone mineral density. Arq Bras Endocrinol Metabol. 2009;53(9):1079-87. doi: 10.1590/ S0004-27302009000900004.

15. Brot C, Vestergaard P, Kolthoff N, Gram J, Hermann AP, Sørensen OH. Vitamin D status and its adequacy in healthy Danish perimenopausal women: relationships to dietary intake, sun exposure and serum parathyroid hormone. Br J Nutr. 2001;86(S1):S97. doi: 10.1079/BJN2001345.

16. Bozkurt S, Alkan BM, Yildiz F, Gümüş S, Sezer N, Ardiçoğlu 2 Özge, et al. Age, sex, and seasonal variations in the serum vitamin D3 levels in a local Turkish population. Arch Rheumatol. 2014;29(1):14-9. doi: 10.5606/tjr.2014.3968.

17. Kroll MH, Bi C, Garber CC, Kaufman HW, Liu D, CastonBalderrama A, et al. Temporal relationship between vitamin D status and parathyroid hormone in the United States. PLoS One. 2015;10(3):e0118108. doi: 10.1371/journal. pone. 0118108 .

18. Vuistiner P, Rousson V, Henry H, Lescuyer P, Boulat O, Gaspoz J-M, et al. A Population-based model to consider the effect of seasonal variation on serum $25(\mathrm{OH}) \mathrm{D}$ and vitamin D status. Biomed Res Int. 2015;2015:1-9. doi: 10.1155/2015/168189.

19. Klingberg E, Oleröd G, Konar J, Petzold M, Hammarsten O. Seasonal variations in serum 25-hydroxy vitamin D levels in a Swedish cohort. Endocrine. 2015;49(3):800-8. doi: 10.1007/ s12020-015-0548-3.

20. Serdar MA, Batu Can B, Kilercik M, Durer ZA, Aksungar FB, Serteser M, et al. Analysis of Changes in Parathyroid Hormone and $25(\mathrm{OH})$ Vitamin D Levels with Respect to Age, Gender and Season: A Data Mining Study. J Med Biochem. 2017;36(1):73-83. doi: 10.1515/jomb-2017-0002.

21. Saraiva GL, Cendoroglo MS, Ramos LR, Araújo LMQ, Vieira JGH, Maeda SS, et al. Prevalência da deficiência, insuficiência de vitamina $\mathrm{D}$ e hiperparatiroidismo secundário em idosos institucionalizados e moradores na comunidade da cidade de São Paulo, Brasil. Arq Bras Endocrinol Metabol. 2007;51(3):437-42. doi: 10.1590/S000427302007000300012.

22. Need AG, Morris HA, Horowitz M, Nordin C. Effects of skin thickness, age, body fat, and sunlight on serum 25-hydroxyvitamin D. Am J Clin Nutr. 1993;58(6):882-5. doi: 10.1093/ajcn/58.6.882.

23. Oliveri MB, Mautalen C, Bustamante L, Gómez García V. Serum levels of 25-hydroxyvitamin D in a year of residence on 
the Antarctic continent. Eur J Clin Nutr. 1994;48(6):397-401.

24. Thomas MK, Lloyd-Jones DM, Thadhani RI, Shaw AC, Deraska DJ, Kitch BT, et al. Hypovitaminosis D in Medical Inpatients. N Engl J Med. 1998;338(12):777-83. doi: 10.1056/ NEJM199803193381201.

25. Mori H, Okada Y, Tanaka Y. Incidence of vitamin D deficiency and its relevance to bone metabolism in Japanese postmenopausal women with type 2 diabetes mellitus. Intern Med. 2015;54(13):1599-604. doi: 10.2169/ internalmedicine. 54.3638 .

26. Arantes HP, Kulak CAM, Fernandes CE, Zerbini C, Bandeira F, Barbosa IC, et al. Correlation between 25-hydroxyvitamin D levels and latitude in Brazilian postmenopausal women: from the Arzoxifene Generations Trial. Osteoporos Int. 2013;24:2707-12. doi: 10.1007/s00198-013-2366-x.

27. Cummings SR, Nevitt MC, Browner WS, Stone K, Fox $\mathrm{KM}$, Ensrud KE, et al. Risk factors for hip fracture in white women. N Engl J Med. 1995;332(12):767-74. doi: 10.1056/ NEJM199503233321202.

28. Bonnick SL, Harris ST, Kendler DL, McClung MR, Silverman SL. Management of osteoporosis in postmenopausal women. Menopause. 2010;17(1):25-54. doi: 10.1097/ gme.0b013e3181c617e6.
29. Mazocco L, Chagas P, Mazocco L, Chagas P. Association between body mass index and osteoporosis in women from northwestern Rio Grande do Sul. Rev Bras Reumatol. 2017;57(4):299-305. doi: 10.1016/j.rbre.2016.10.002.

30. Sugiyama T, Yamaguchi A, Kawai S. Effects of skeletal loading on bone mass and compensation mechanism in bone: a new insight into the "mechanostat" theory. J Bone Miner Metab. 2002;20(4):196-200. doi: 10.1007/s007740200028.

31. Rodrigues Filho EA, Santos MAM, Silva ATP, Farah BQ, Costa MC, Campos FACS, et al. Relation between body composition and bone mineral density in young undregraduate students with different nutritional status. Einstein (São Paulo). 2016;14(1):12-7. doi: 10.1590/S1679-45082016AO3569.

32. Lips P, Obranr KJ. The pathogenesis and treatment of hip fractures. Osteoporosis Int. 1991;1:218-31. Available from: https://link.springer.com/content/pdf/10.1007/BF03187466. pdf.

33. Lips P. Vitamin D deficiency and osteoporosis: the role of vitamin $\mathrm{D}$ deficiency and treatment with vitamin $\mathrm{D}$ and analogues in the prevention of osteoporosis-related fractures. Eur J Clin Invest. 1996;26(6):436-42. https://doi.org/10.1046/ j.1365-2362.1996.176290.x

34. Stephen AB, Wallace WA. The management of osteoporosis. J Bone Joint Surg Br. 2001;83(3):316-23. 